\title{
Assessment of effects of the genetic polymorphisms on soccer athletes
}

\author{
W.C.F. Menezes 1 , T.C.V. Gingozac ${ }^{2}$, L.A.S. Oliveira ${ }^{2}$, G.R. Pedrino ${ }^{3}$, \\ Y.M.F. Ternes ${ }^{4}$, R.S. Santos ${ }^{1,5}$ and A.A.S. Reis ${ }^{16^{6}}$ \\ ${ }^{1}$ Laboratório de Patologia Molecular, Instituto de Ciências Biológicas, \\ Universidade Federal de Goiás, Goiânia, GO, Brasil \\ ${ }^{2}$ Faculdade do Esporte, Universidade Estadual de Goiás, Goiânia, GO, \\ Brasil \\ ${ }^{3}$ Departamento de Fisiologia, Instituto de Ciências Biológicas, Universidade \\ Federal de Goiás, Goiânia, GO, Brasil \\ ${ }^{4}$ Departamento de Saúde Pública, Instituto de Patologia Tropical e Saúde \\ Pública, Universidade Federal de Goipas, Goiânia, GO, Brasil \\ ${ }^{5}$ Departamento de Ciências da Natureza, Unidade Acadêmica Especial de \\ Ciências Humanas,Universidade Federal de Goiás, Goiás, GO, Brasil \\ ${ }^{6}$ Departamento de Bioquímica e Biologia Molecular, Instituto de Ciências \\ Biológicas, Universidade Federal de Goiás, Goiânia, GO, Brasil
}

Corresponding author: A.A.S. Reis

E-mail: angeladamski@gmail.com

Genet. Mol. Res. 18 (2): gmr18192

Received November 29, 2018

Accepted May 07, 2019

Published May 13, 2019

DOI http://dx.doi.org/10.4238/gmr18192

\begin{abstract}
Several genes have been identified as important in athletic performance. The angiotensin-converting enzyme (ACE) insertion/deletion (I/D) polymorphism has been related to improvements in performance and exercise duration. However, there are large divergences among studies on the influence of ACE I/D polymorphism on physical performance. Other studies have demonstrated that strenuous exercise generally overloads the endogenous antioxidant system's capacity, leading to oxidative damage to muscles and other tissues in athletes. Few studies have reported significant associations between glutathione S-transferase (GST) deletion polymorphisms and resistance performance in athletes. We examined the effects of ACE I/D and GST deletion polymorphisms in young soccer players in central Brazil. We included 65 soccer players from under-20 (18 to 20 years old) soccer
\end{abstract}


teams and 60 non-athletes in a case-control study. All subjects had their physical performance investigated through the Running-based Anaerobic Sprint Test (RAST). Genotyping was performed using real-time PCR. We found that the I/D genotype was significantly more frequent in athletes compared with the non-athletes. Individuals with the ID and DD genotypes had an approximately 3.13-fold and 4.37-fold increase in strength and power-orientated performance, respectively. The DD genotype in athletes presented RAST-test results considered as excellent to good when compared with non-athletes. When we examined a possible association of the GST deletion polymorphisms and RAST test, we found no influence on athletic performance. We did find association between ACE I/D polymorphism and athletic performance. However, there was no association between GST polymorphisms and risk of oxidative damage in the muscles. Our findings may help to select young players with the most favorable genetic potential to succeed as soccer athletes.

Key words: ACE I/D polymorphism; GSTT1/GSTM1 deletion polymorphisms; RAST test; Young soccer athletes

\section{INTRODUCTION}

Several genes have been identified as relevant for improving athletic performance. In the case-control GENATHLETE study, single nucleotide polymorphisms (SNPs) in several candidate genes were investigated, but none provided strong evidence for differences in allele and genotype frequencies between elite athletes compared with control subjects (Rankinen et al., 2016). Previously published reports have focused on differences in allele frequencies between athletes and non-athletes in some polymorphisms, such as angiotensin I converting enzyme (ACE) alleles (Rankinen et al., 2000; Rankinen et al., 2016). This polymorphism became the first genetic element shown to substantially impact human physical performance (Grealy et al., 2015; Papadimitriou et al., 2018).

The renin-angiotensin system (RAS) is an endocrine regulator that also influences local tissues and cells, where it serves a variety of functions. The ACE insertion/deletion (ACE I/D, rs 1799752) polymorphism has been related to improvements in performance and exercise duration in various populations (De Mello Costa et al., 2012; Ma et al., 2013; Weyerstra et al., 2017). The I allele, represented by a 287 bp insertion, has been consistently linked to endurance-orientated events, lower serum levels of ACE, and improved performance in endurance sports. The deleted form of the variant (D allele) has been associated with higher circulating and tissue ACE activity, promoting strength, and powerorientated performance (Nazarov et al., 2001; Micheli et al., 2011; Almeida et al., 2012; Jacob et al. 2018).

Many athletes and individuals participating in regular exercise programs consume antioxidant supplements to avoid enhanced production of reactive oxygen species (ROS) in response to exercise (Akimoto et al., 2010; Miranda-Vilela et al., 2011). However, strenuous exercise (undertaken above the habitual intensity of effort), or training with a very elevated cardiac frequency will generally overload the endogenous antioxidant system's capacity, leading to an increase in plasma lipid peroxidation plus oxidative damage to 
muscles and other tissues (Vecchio, 2017). Some polymorphisms have also been implicated in changed lipid levels, immune reactivity, and biotransformation of many substances, such as products of oxidative stress and athletic performance (Miranda-Vilela et al., 2011).

Individual genetic differences may affect the antioxidant response and influence athlete performance and health. Glutathione S-transferase (GST) is a multigenic superfamily of phase II detoxification enzymes. GST plays a crucial role in cellular protection against oxidative stress by promoting detoxification. Moreover, this group of genes mediate the conjugation of reduced glutathione to electrophilic species, leading to the elimination of toxic compounds and thus favoring an antioxidant response (Pinheiro et al., 2017; Zarebska et al., 2017). Furthermore, GSTM1 and GSTT1 deletion polymorphisms could influence performance induced by effort and resistance (Akimoto et al., 2010; Miranda-Vilela et al., 2011).

There are significant divergences among studies reporting the impact of ACE I/D polymorphism on physical performance. However, few studies have been published on the association between the GSTT1/GSTM1 deletion polymorphisms and athletic performance. This first study on the Central Brazilian population was designed to provide more information about the effects of the ACE I/D and GSTT1/GSTM1 polymorphisms on soccer players and their performance.

\section{MATERIAL AND METHODS}

In our study, we investigated 65 young soccer players from two under-20 (18 to 20 years old) soccer teams in Goiás, Brazil. Our control group was composed of 60 nonathletes from a fitness center in the metropolitan region of Goiânia, Goiás, Brazil. This research was approved by the Research Ethics Committee of the Federal University of Goiás (UFG), Brazil. This study was conducted following the Ethical Principles for Medical Research Involving Human Subjects of the World Medical Association and the Declaration of Helsinki.

All procedures followed in this study meet the ethical standards in Sport and Exercise Science Research and were approved by appropriate local ethics committees. Written informed consent was obtained from all participants of this research. We set the inclusion criteria according to The STrengthening the REporting of Genetic Association studies (STREGA) guidelines for improved reporting of genetic association studies (Little et al., 2009).

Each subject involved in the study had their physical performance evaluated using the Running-based Anaerobic Sprint Test (RAST). The RAST test was used for collecting indicators related to speed, strength, and power-oriented performance. In this test, all individuals of the study were asked to run a distance of $35 \mathrm{~m}$ six times at their maximum possible speed, with rest periods of 10 s between each of the six runs.

Peripheral blood samples were collected in heparinized tubes, and genomic DNA was extracted using the DNA PureLink ${ }^{\circledR}$ kit (Invitrogen by Life Technologies, USA). The DNA samples were stored in a freezer $\left(-80^{\circ} \mathrm{C}\right)$. To determine ACE polymorphism, the Insertion (I) and Deletion (D) alleles were analyzed by a real-time PCR assay, followed by melting curve analysis. The primers and the thermocycling conditions were applied as previously described (Lin et al., 2001). 
The GSTM1 and GSTT1 deletion polymorphisms were determined through a multiplex real-time PCR, followed by melting curve analysis, as previously described (Pinheiro et al., 2013). The homozygous deletion was designated as null genotype (complete deletion of both alleles), and the positive genotype had at least one wild-type allele.

\section{Statistical analysis}

The chi-square test was used to compare the genotype frequencies. Allelic frequencies were tested for Hardy-Weinberg equilibrium. The odds ratio (OR) and 95\% confidence interval $(\mathrm{CI})$ provided a measure of the strength of association to assess the impact of young soccer players' genotype on physical performance. The prevalence ratio was used to estimate the association between the RAST test, ACE genotypes, and GSTs polymorphisms. Logistic regression was used to determine the relationship between the RAST test and the polymorphisms. A P-value of less than 0.05 was considered statistically significant. All statistical analyses were conducted using the STATA (v. 13.0).

\section{RESULTS}

Genotyping was carried out successfully in all individuals. The genotype frequencies are shown in Table 1 . In the young soccer players, the frequency of the I allele was $49 \%$, whereas for the control group it was $60 \%$. In addition, the frequency for the D allele was 51 and $40 \%$ for young athletes and non-athletes, respectively.

The ID and DD genotypes were significantly more frequent in athletes than in the control group, with $\mathrm{P}=0.01$ and $\mathrm{P}=0.03$, respectively. For the GSTM1/GSTT1 frequency, the GSTM1 genotype did not reach statistical significance in differentiating individuals with null and positive genotypes $(\mathrm{P}=0.065)$. For the GSTT1 genotype, we did not observe significant differences between individuals with null and positive genotypes $(\mathrm{P}=0.281$, Table 1).

The high frequency of the $\mathrm{D}$ allele in young soccer players demonstrated that this polymorphism could influence physical performance. The DD individuals presented a 4.37fold increase in athletic performance in relation to the other ID genotypes $(P=0.039)$. In addition, the ID genotype was associated with a 3.13-fold increase in athletic performance. Conversely, for the GST polymorphism, significant differences were not observed for any genotype (Table 1).

Table 1. The distribution of genotypes in young soccer players (YSP) and non-athletes (NA).

\begin{tabular}{|c|c|c|c|c|c|c|c|}
\hline Gene & Genotype & YSP n & NA n & $\chi^{2}$ & $\mathbf{P}$ & OR (CI 95\%) & $\mathbf{P}$ \\
\hline \multirow[t]{3}{*}{$\mathrm{ACE}$} & II & 7 & 17 & & & 1.00 (Reference) & \\
\hline & ID & 49 & 38 & 5.54 & $0.018 *$ & $3.13(1.1790-8.3179)$ & $0.022 *$ \\
\hline & DD & 9 & 5 & 4.47 & $0.034 *$ & $4.37(1.0742-17.7900)$ & $0.039 *$ \\
\hline \multirow[t]{2}{*}{ GSTM1 } & Positive & 49 & 36 & & & 1.00 (Reference) & \\
\hline & Null & 16 & 24 & 3.39 & 0.065 & $0.48(0.2279-1.0526)$ & 0.067 \\
\hline \multirow[t]{2}{*}{ GSTT1 } & Positive & 46 & 37 & & & 1.00 (Reference) & \\
\hline & Null & 19 & 23 & 1.15 & 0.281 & $0.66(0.3151-1.4011)$ & 0.282 \\
\hline
\end{tabular}

Young soccer players (YSP) and non-athletes (NA). $\chi^{2}=$ Chi-Square; OR $=$ odds ratio, $\mathrm{CI}=$ confidence interval, ${ }^{*} \mathrm{P}<0.05$ denotes significance. 
We quantified the strength of the association between the young soccer players' genotype and performance on the RAST test through an OR and a 95\% confidence interval (Table 2). The performance was compared with the control group (non-athletes) and adjusted for confounding factors. The athletes with the DD genotype had excellent to good results and a 9.33-fold increase in performance when compared to the control group ( $\mathrm{P}=$ 0.010). Furthermore, the $\mathrm{D}$ allele was associated with a 4.14-fold increase in excellent to good results in the RAST test for ID individuals $(\mathrm{P}=0.04)$. Acceptable to weak results for the RAST test demonstrated that the I allele had no influence on performance.

For the association between the GSTM1 and GSTT1 genotypes and the RAST test, there was no apparent influence of these polymorphisms on athletic performance. However, for the combination genotypes, the data showed a tendency for GSTM1/T1 (+/-) to be associated with excellent to good results in the RAST test. However, there was the increase in physical performance, but was not significant $(\mathrm{OR}=0.33 ; \mathrm{CI}=0.100-1.109 ; \mathrm{P}=0.073$, Table 2).

Table 2. Distribution of ACE and GSTM1/GSTT1 (combination) genotypes; and the association analysis for the RAST test in young soccer players (YSP) and non-athletes (NA).

\begin{tabular}{|c|c|c|c|c|c|c|c|}
\hline RAST ACE & & YSP n & NA n & $\chi^{2}$ & $\mathbf{P}$ & OR $(95 \% \mathrm{CI})$ & $\mathbf{P}$ \\
\hline \multirow{3}{*}{ Excellent to Good } & II & 3 & 14 & & & 1.00 (Reference) & \\
\hline & ID & 24 & 27 & 4.60 & $0.031^{*}$ & $4.14(1.062-16.208)$ & $0.040^{*}$ \\
\hline & $\mathrm{DD}$ & 8 & 4 & 7.18 & $0.007 *$ & $9.33(2.014-76.874)$ & $0.010^{*}$ \\
\hline \multirow{3}{*}{ Acceptable to Weak } & II & 4 & 3 & & & 1.00 (Reference) & \\
\hline & ID & 25 & 11 & 0.40 & 0.525 & $1.70(0.325-8.933)$ & 0.528 \\
\hline & DD & 1 & 1 & 0.03 & 0.858 & $0.75(0.032-17.507)$ & 0.857 \\
\hline RAST GST & M1/T1 & YSP n & NA n & $\chi^{2}$ & $\mathbf{P}$ & OR $(95 \%$ IC) & $\mathbf{P}$ \\
\hline \multirow{4}{*}{ Excellent to Good } & $+/+$ & 18 & 13 & & & 1.00 (Reference) & \\
\hline & $+/-$ & 6 & 13 & 3.31 & 0.068 & $0.33(0.100-1.109)$ & 0.073 \\
\hline & $-1+$ & 7 & 13 & 2.58 & 0.107 & $0.38(0.121-1.245)$ & 0.111 \\
\hline & $-1-$ & 2 & 7 & 3.58 & 0.058 & $0.20(0.037-1.159)$ & 0.073 \\
\hline \multirow{4}{*}{ Acceptable to Weak } & $+/+$ & 14 & 6 & & & 1.00 (Reference) & \\
\hline & $+/-$ & 6 & 2 & 0.07 & 0.792 & $1.28(0.199-8.294)$ & 0.791 \\
\hline & $-1+$ & 5 & 3 & 0.14 & 0.701 & $0.71(0.122-3.994)$ & 0.701 \\
\hline & $-1-$ & 7 & 1 & 0.93 & 0.333 & $3.00(0.0299-30.020)$ & 0.350 \\
\hline
\end{tabular}

RAST $=$ Running-based Anaerobic Sprint Test, $\boldsymbol{\chi}^{2}=$ Chi-Square; OR=odds ratio, CI= Confidence Interval, $* \mathrm{P}<0.05$ denotes statistical significance.

In the logistic regression model, we evaluated the association between the RAST test and the polymorphisms (Table 3). It was not possible to observe any association between the analyzed variables.

Table 3. RAST test comparison between the polymorphisms.

\begin{tabular}{lcc}
\hline Polymorphism & RAST PR* $($ CI 95\%) & P \\
\hline GST (M1/T1) & $1.19(0.879-1.632)$ & 0.77 \\
Genotype ID/DD and II & $1.19(0.879-1.632)$ & 0.68 \\
\hline
\end{tabular}

RAST= Running-based Anaerobic Sprint Test; CI = confidence interval. * Prevalence Rate 


\section{DISCUSSION}

Human athletic performance is a highly complex phenotype that could be considered as a multifactorial, polygenic trait. In general, the association of genetic polymorphisms with sporting prowess is recognized in studies of elite athletes drawn from a single sport (Pitsiladis et al., 2013). Notably, the ACE I/D polymorphism has been reported variably in different sports modalities (Puthucheary et al., 2011). However, some studies have shown a significant association between these genomic features and sports performance (Papadimitriou et al., 2018). For GSTM1/GSTT1 deletion polymorphisms, there are few studies relating these polymorphisms to athletic performance.

In our findings, for both groups, there was a greater frequency of the ID genotype of the ACE I/D polymorphism, such as in the frequency of the GSTM1/GSTT1 positive genotype. However, the DD genotype and GSTM1/GSTT1 null genotype were less frequent than other genotypes in this population.

The finding of the $\mathrm{D}$ allele in young soccer players demonstrated that this polymorphism could influence physical performance. The DD and ID individuals showed an increased potential for improved sporting performance. These findings are in agreement with other reports (Gineviciene et al., 2016; Ribas et al., 2017; Weyerstra et al., 2017; Jacob et al., 2018) and can be justified by the high degree of heterogeneity found in the Brazilian population (Santos et al., 2015).

In a sporting context, according to Jacob et al., (2018), the frequency of I alleles presumably increases with racing distance elite Caucasian runners and swimmers. Therefore, the insertion allele appears to be an advantageous genotype for endurance sports. Athletes with this allele tend to have an increased aerobic capacity and better performance in a range of endurance sports (Thaylor et al., 1999). The ACE allele deletion has commonly been associated with sporting performance dependent on strength and power.

This particular allele is related to circulating plasma levels of ACE, with a DD genotype resulting in significantly higher levels of ACE compared to the II genotype (Ostrander et al, 2009). Our study suggests that individuals with DD and ID genotypes are more predisposed to greater physical strength and power due to the presence of the D allele. Concordantly, our findings are in agreement with the studies made in Australia and Turkey (Ulucan et al., 2015; Jacob et al., 2018). In a Serbian study with female soccer players, there was a high prevalence of the DD genotype, suggesting that individuals with this genotype tend to perform better in soccer (Jeremic et al., 2019).

The RAST test has been used as an indicator of anaerobic power and oxygen uptake profile as it requires a greater contribution from aerobic metabolism (Keir et al., 2013). The main finding of our study is that ACE polymorphism was associated with anaerobic performance. We demonstrated that in the RAST test, athletes with the DD genotype presented excellent to good performance, indicating that the DD genotype may be more beneficial for anaerobic power $(\mathrm{OR}=9.33, p=0.010)$. A meta-analysis demonstrated a significant association of the ACE D allele with elite power athletes' status, suggesting that the ACE D allele can be considered a power allele (Weyerstra et al., 2017).

On the other hand, there is a higher level of ACE activity for D allele carriers, resulting in an increase in both angiotensin II and metabolism of bradykinin, which, in addition to blood pressure regulation, has a significant impact on metabolic processes (De Mello Costa et al., 2012). In a Brazilian study, it was found that the ACE - DD genotypic 
distribution in wrestlers was favorable to activities involving strength and power. The authors reported that the DD genotype was more predominant in wrestlers compared with controls (45.7 vs. $32.2 \%$, respectively). This study also suggested that the more predisposed to fight sports are individuals with ACE gene alleles related to strength and muscle power (Ribas et al., 2017). Thus, based on the role of ACE I/D polymorphism in anaerobic power, strength, and muscle power, we speculate that determining ACE genomic status could facilitate identifying athletes with optimal performance in distinct soccer positions.

On the other hand, several investigations have provided evidence that exercise can either positively or negatively affect oxidative status based on training load, training specificity, and the basal level of training (Akimoto et al., 2010). Indeed, studies have shown that even anaerobic exercise (e.g., high-intensity training) can produce similar levels of oxidative stress (Miranda-Vilela et al., 2011). The degree of oxidative damage and the time course for elevation in oxidative stress markers - during and following both acute aerobic and anaerobic exercise - are dependent on the type, intensity, volume, and duration of muscle contraction. Moreover, the oxidative mechanisms are also influenced by gender, age, individual fitness levels, and nutritional status, leading to differences in the oxidative status between athletes in different sporting disciplines (Vecchio et al., 2017). However, in our study, there was no association between the GSTM1/GSTT1 deletion polymorphisms and better athletic performance.

This first study of the central Brazilian population, in which we investigated gene polymorphisms and exercise-induced oxidative stress, DNA damage was dependent on the interaction between the combinations of specific genotypes. These results indicate that, in runners, other polymorphisms may influence performance but not GSTM1/GSTT1 deletion or ACE polymorphism (Akimoto et al., 2010). Our study is also the first to relate genetic polymorphisms to athletic performance in young Brazilian soccer players. Our findings are in keeping with those of Akimoto et al. (2010), who reported that GSTM1/GSTT1 deletion did not significantly affect performance.

Zarebska et al. (2017) emphasized that the G allele of the GSTP1 gene (c.313AG single nucleotide polymorphism) is associated with improved endurance performance. These observations could support the hypothesis that the GSTP1 G allele improves exercise performance by better elimination of exercise-induced reactive oxygen species (Pitsiladis et al., 2013). In addition, our study also investigated the role of the GSTM1/GSTT1 polymorphisms in young soccer players' performance, but the results did not reach statistical significance.

Soccer is an intermittent sport characterized by approximately 1200 acyclical and unpredictable changes in activity (every 3 to 5s). These typically involve as many as 40 sprints, more than 700 turns, seven jumps, and up to 40 tackles. In addition, the game requires other intense actions such as decelerations, kicking, dribbling, and tackling. All these efforts exacerbate the physical strain imposed on players and contribute to making football a physiologically demanding sport (Iaia et al., 2009), which involves both aerobic and anaerobic metabolism (Vecchio et al., 2017). We found that the D allele and DD genotype predispose the individual to greater strength and muscle power. However, GSTM1/GSTT1 deletion polymorphisms should be further investigated with regards to the oxidative stress response in high-intensity training. Athletic performance is a highly complex, multifactorial, and polygenic trait. These results could be employed for identifying young athletes with the greater genetic potential to succeed in soccer 
performance. Moreover, these athletes could undergo customized training based on their genotypes.

\section{CONCLUSIONS}

The first study in the Central Brazilian population that investigated the influences of genes polymorphisms, described the association on the ACE genotypes, and it's might help select young with the most favorable genetic potential to succeed such as soccer athletes. On the other hand, the GST polymorphism indicated no associations for risk oxidative damage muscle.

\section{ACKNOWLEDGMENTS}

The authors would like to thank the Goiás Sports Club, Vila Nova Sports Club, and Flectir Fitness Center for providing samples for this study. Funding for this study was financed in part by the Coordination for the Improvement of Higher Education Personnel (CAPES - Brazil) - Finance Code 001- grants AUXPE 1665/2016.

\section{CONFLICTS OF INTEREST}

The authors have no conflicts of interest that are relevant to the contents of this paper.

\section{REFERENCES}

Akimoto AK, Miranda-Vilela AL, Alves PCZ, Pereira LCS, et al. (2010). Evaluation of gene polymorphisms in exercise-induced oxidative stress and damage. Free Radic. Res. 44: 322-31.

Almeida JA, Boullosa DA, Pardono E, et al. (2012). The Influence of ACE Genotype on Cardiorespiratory Fitness of Moderately Active Young Men. Arq. Bras. Cardiol. 98: 315-20.

de Mello Costa MF and Slocombe R (2012). The use of angiotensin-I converting enzyme I/D genetic polymorphism as a biomarker of athletic performance in humans. Biosensors. 2: 396-404.

Iaia FM, Rampinini E and Bangsbo J (2009). High-Intensity Training in Football. Int J Sports Physiol Perform. 4: 291306.

Gineviciene V, Jakaitiene A, Aksenov MO, Aksenova AV, et al. (2016). Association analysis of ACE, ACTN3, and PPARGC1A gene polymorphisms in two cohorts of European strength and power athletes. Biol. Sport. 33: 199206.

Grealy R, Herruer J, Smith CL, Hiller D, et al. (2015). Evaluation of a 7-Gene Genetic Profile for Athletic Endurance Phenotype in Ironman Championship Triathletes. PLoS One.. 10: e0145171.

Jacob Y, Spiteri T, Hart NH and Anderton RS (2018). The Potential Role of Genetic Markers in Talent identification and Athlete Assessment in Elite Sport. Sports. 6: e88.

Jeremic D, Macuzic I, Vulonic M and Stevanovic J (2019). ACE/ACTN3 genetic polymorphisms and athletic performance of female soccer players. Bras. Med. Esporte. 25:35-39.

Keir DA, Thériault F and Serresse O (2013). Evaluation of the running-based anaerobic sprint test as a measure of repeated sprint ability in collegiate-level soccer players. J. Strength. Cond. Res. 27: 1671-8.

Lin M-H, Tseng C-H, Tseng CC, Huang C-H, et al. (2001). Real-time PCR for rapid genotyping of angiotensinconverting enzyme insertion/deletion polymorphism. Clin. Biochem. 34: 661-6.

Little J, Higgins JPT, Ioannidis JPA, Moher D, et al. (2009). Strengthening the reporting of genetic association studies (STREGA): an extension of the STROBE Statement. PLoS Medicine. 6: e1000022.

Ma F, Yang Y, Li Y, Zhou F, et al. (2013). The Association of Sport Performance with ACE and ACTN3 Genetic Polymorphisms: A Systematic Review and Meta-Analysis. PLoS One. 8: e54685.

Micheli ML, Gulisano M, Morucci G, et al. (2011). Angiotensin-converting enzyme/vitamin d receptor gene polymorphisms and bioelectrical impedance analysis in predicting athletic performances of Italian young soccer players. J. Strength. Cond. Res. 25: 2084-91. 
Miranda-Vilela AL, Lordelo GS, Akimoto AK, et al. (2011). Genetic polymorphisms influence runners' responses to the dietary ingestion of antioxidant supplementation based on pequi oil (Caryocar brasiliense Camb.): a before-after study. Genes Nutr. 6: 369-395.

Nazarov IB, Woods DR, Montgomery HE, Shneider OV, et al. (2001). The angiotensin-converting enzyme I/D polymorphism in Russian athlete. Eur. J. Hum. Genet. 9: 797-801.

Ostrander EA, Huson HJ and Ostrander GK (2009). Genetics of athletic performance. Annu. Rev. Genom. Human Genet. 10: 407-429.

Papadimitriou ID, Lockey SJ, Voisin S, Herbert AJ, et al. (2018). No association between ACTN3 R577X and ACE I/D polymorphisms and endurance running times in 698 Caucasian athletes. BMC. Genomics.19: 13.

Pinheiro DS, Rocha Filho CS, Mundim CA, de Marco Júnior P, et al. (2013). Evaluation of Glutathione S-Transferase GSTM1 and GSTT1 Deletion Polymorphisms on Type-2 Diabetes Mellitus Risk. PLoS One. 8: e76262.

Pinheiro DS, dos Santos RS, de Brito RB, Cruz AHS, et al. (2017). GSTM1/GSTT1 double-null genotype increases the risk of treatment-resistant schizophrenia: A genetic association study in Brazilian patients. PLoS One. 12: e0183812

Pitsiladis Y, Wang G, Wolfarth B, et al. (2013). Genomics of elite sporting performance: what little we know and necessary advances. Br. J. Sports Med. 47: 550-5.

Puthucheary Z, Skipworth JRA, Rawal J, et al. (2011). The ACE gene and human performance. Sport Med. 41: 433-48

Rankinen T, Wolfarth B, Simoneau JA, Maier-Lenz D, et al. (2000). No association between the angiotensin-converting enzyme ID polymorphism and elite endurance athlete status. J. App. Physiol. 88: 1571-5.

Rankinen T, Fuku N, Wolfarth B, et al. (2016). No Evidence of a Common DNA Variant Profile Specific to World Class Endurance Athletes. Plos One. 11: e0147330.

Ribas MR, Oliveira Neto ZC, Salgueirosa F and Fernandes P (2017). Association of ACTN3 R577X and ACE I/D polymorphisms in Brazilians wrestlers. Rev. Bras. Med. Esporte. 23: 469-472.

Santos RV, da Silva GO and Gibbonc O (2015). Pharmacogenomics, human genetic diversity and the incorporation and rejection of color/race in Brazil. Biosocieties. 10: 48-69.

Thaylor RR, Mamotte CD, Fallon K and van Bockxmeer FM (1999). Elite athletes and the gene for angiotensinconverting enzyme. J. Appl. Physiol. 87: 1035-7.

Ulucan K, Sercan C and Biyikli T (2015). Distribution of angiotensin-1 converting enzyme insertion/deletion and $\alpha$ actinin-3 codon 577 polymorphisms in Turkish male soccer players. Genet. Epigenet. 7: 1-4.

Vecchio M, Currò M, Trimarchi F, Naccari S, et al. (2017). The Oxidative Stress Response in Elite Water Polo Players: Effects of Genetic Background. Biomed. Res. Int. 2017: 7019694.

Weyerstra J, Stewart K, Wesselius A and Zeegers M (2017). Nine genetic polymorphisms associated with power athlete status - A Meta-Analysis. J. Sci. Med. Sport. 2: 213-220.

Zarebska A, Jastrzebski Z, Ahmetov II, Zmijewski P, et al. (2017). GSTP1 c.313AG polymorphism in Russian and Polish athletes. Physiol. Genomics. 49: 127-131. 